\title{
Endobronchial ultrasound-guided transbronchial lung biopsy in solitary pulmonary nodules and peripheral lesions
}

\author{
F.J.F. Herth*, A. Ernst", H.D. Becker*
}

Endobronchial ultrasound-guided transbronchial lung biopsy in solitary pulmonary nodules and peripheral lesions. F.J.F. Herth, A. Ernst, H.D. Becker. CERS Journals Ltd 2002

ABSTRACT: Transbronchial biopsy (TBBX) for peripheral lung lesions is usually performed with the help of fluoroscopy, but the yield varies widely. This feasibility study aimed to assess the ability of endobronchial ultrasound (EBUS) to provide imaging guidance for TBBX.

In a prospective study, 50 consecutive patients referred for TBBX for peripheral lesions underwent fluoroscopy-guided and EBUS-guided TBBX in random order. Diagnostic yields were compared for both modalities and feasibility was assessed for EBUS.

Diagnostic material was obtained in $80 \%$ of patients with EBUS and $76 \%$ of patients with fluoroscopy. There was a nonsignificant trend for EBUS to be better than fluoroscopy for lesions $<3 \mathrm{~cm}$ in diameter. Four lesions could not be visualised with EBUS. There were no significant complications associated with the use of EBUS.

Endobronchial ultrasound-guided transbronchial biopsy is feasible. It appears to be at least equivalent to fluoroscopy without the accompanying radiation exposure. Further large-scale studies are indicated to assess the possible role of endobronchial ultrasound as a potential imaging method of choice for the biopsy of peripheral lung lesions. Eur Respir J 2002; 20: 972-974.
*Dept of Interdisciplinary Endoscopy, Thoraxklinik Heidelberg, Germany and \# Interventional Pulmonology, Division of Pulmonary and Critical Care Medicine, Beth Israel Deaconess Medical Center, Harvard Medical School, Boston, MA, USA.

Correspondence: F.J.F. Herth, Dept of Interdisciplinary Endoscopy, Amalienstr. 5, D-69126 Heidelberg, Germany. Fax: 496221396246

E-mail: f@herth.net

Keywords: Biopsy, bronchoscopy, endobronchial ultrasound, lung mass, solitary peripheral nodule

Received: January 22002

Accepted after revision: May 272002
Bronchoscopy has been used for over $30 \mathrm{yrs}$ in the evaluation of solitary pulmonary nodules (SPN) and peripheral lesions of the lung. Flexible bronchoscopy (FB) is frequently performed in patients with such lesions to establish a diagnosis. Usually, the procedure is performed as a transbronchial biopsy (TBBX) with fluoroscopy guidance. The complication rate is generally low, but there is certainly radiation exposure to patients and staff. Additionally, the diagnostic yield of FB has a wide variability $(18-75 \%)[1,2]$. Previous studies on SPN and peripheral lesions have consistently shown that the size of the lesion and its location influence the diagnostic accuracy of bronchoscopy [1, $3-5]$. Therefore, in many institutions, patients undergo primary surgical biopsy procedures, such as videoassisted thoracic surgery. In order to save patients the need for operative procedures, new imaging and guidance technology would be desirable. The present study evaluated endobronchial ultrasound (EBUS) and its ability to guide TBBX for peripheral lung lesions. EBUS has received increasing attention in other bronchoscopic procedures, such as transbronchial needle aspiration (TBNA) $[4,6]$. This is the first report on the use of EBUS for guidance of transbronchial biopsies.

\section{Methods}

\section{Patients and methods}

In a prospective crossover study from November 2000-February 2001, 50 consecutive patients with peripheral lesions referred for diagnostic bronchoscopy were enrolled. All chest computed tomographs were reviewed and the size of the lesions were recorded by their longest diameter. After written informed consent, patients underwent bronchoscopy. Procedures were performed under general anaesthesia or conscious sedation in standard fashion. A variety of fibreoptic bronchoscopes (models BF 1T-30, BF 1T 40 and BF XT 20; Olympus Co., Tokyo, Japan) were used. Biopsies were performed with regular disposable biopsy forceps. Forceps were changed between EBUS and fluoroscopic examinations to avoid cellular cross contamination. After complete inspection of the bronchial tree, including the subsegmental bronchi, TBBX was performed sequentially under EBUS and fluoroscopic guidance in random order in every patient. Fluoroscopy was provided using a monoplanar C-arm (Suprer 50 CP, Philipps Co., Amsterdam, the Netherlands). EBUS was performed with a flexible probe and processor unit (UM-3R, UM-4R, US2020R, Olympus) as described below. Histological diagnosis of fibrosis was considered nondiagnostic. The histological results were compared for the two methods.

\section{Technique of transbronchial lung biopsy}

Fluoroscopy. After fluoroscopic localisation of the lesion, the forceps (FB-20C, Olympus) were advanced towards the lesion. The cups of the forceps were then 


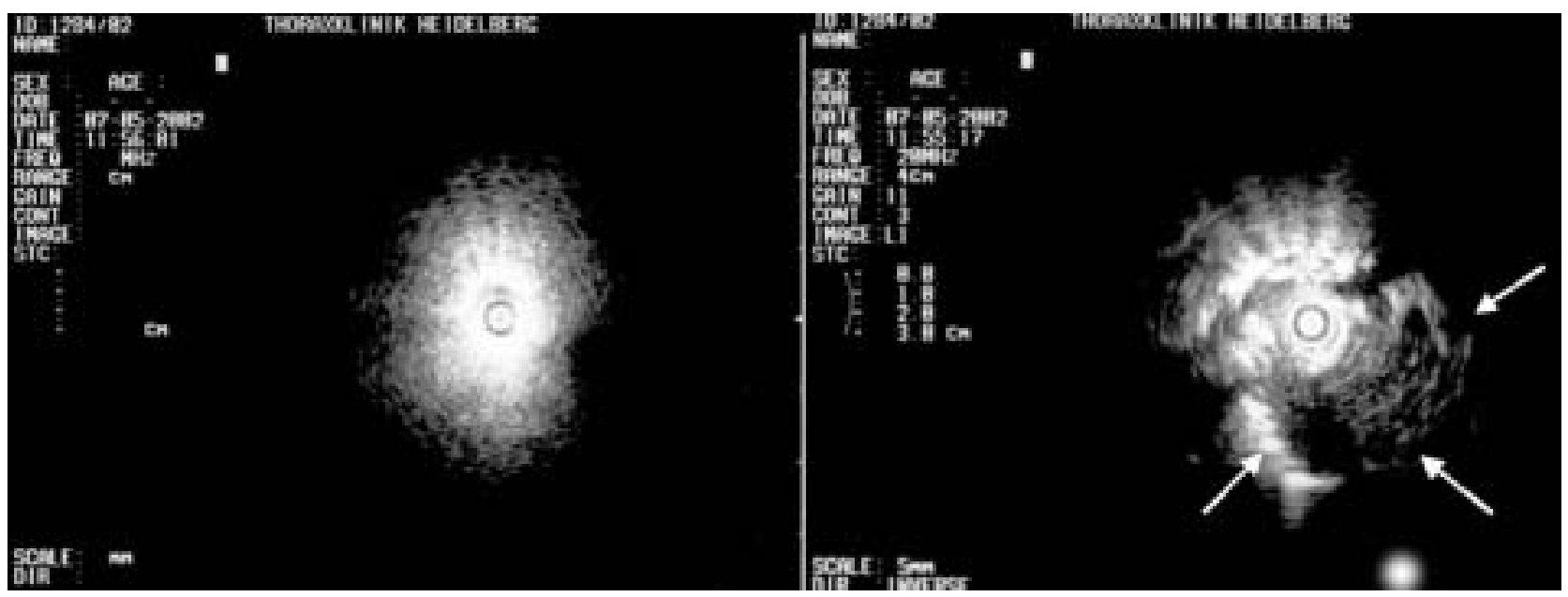

Fig. 1.-Endobronchial ultrasound (EBUS) image of an air-filled peripheral lung, which is typically "snowstorm-like" (to the left). The image obtained when entering solid tissue in the periphery is shown to the right. A definite hypoechogeneic signal can be appreciated (outlined by arrows).

opened and advanced into the lesion. A minimum of four specimens were taken.

Endobronchial ultrasound. The probes were inserted like the forceps into the different bronchi, where the lesion was suspected. In contrast to the "snowstormlike" whitish image of air-containing lung tissue, solid lesions appear darker and more homogenous. Usually, they are well differentiated against the lung tissue by a bright border due to the difference in impedance (fig. 1). After localising the lesion, the probe was removed from the biopsy channel and the forceps introduced into the corresponding subsegmental bronchus and $\geqslant 4$ biopsies were performed.

\section{Statistical analysis}

The Spearman rank correlation for nonparametric samples was used to correlate the different classifications with the histological results. Data are presented as mean $\pm \mathrm{SD}$.

\section{Results}

Thirty-seven males and 13 females with an average age of $62.5 \pm 10.5 \mathrm{yrs}$ (range 25-81 yrs) (table 1) were examined. Forty-three patients $(86 \%)$ were smokers. The mean diameter of the lesion was $3.31 \pm 0.92 \mathrm{~cm}$, (range $2-6 \mathrm{~cm}$ ). The localisation of the abnormality was the right middle lobe in four patients $(8 \%)$, the left upper lobe in $11(22 \%)$, the right upper lobe in 27 $(54 \%)$, the left lower lobe in six $(12 \%)$ and the right lower lobe in two $(4 \%)$. The mean number of specimens obtained was $4.34 \pm 0.55$ under EBUS guidance and 4.56 \pm 0.61 under fluoroscopy (statistically not significant).

In EBUS-guided TBBX diagnosis was established in 40 patients $(80 \%)$. In four cases, the lesion could not be localized; all of these were localised in the right upper lobe. Under fluoroscopic guidance, diagnosis could be established in 38 patients $(76 \%)$. All nodules could be localised by fluoroscopy. All patients, in whom a definite diagnosis could not be established, underwent a surgical procedure. Tables 1 and 2 show the yield by location of the lesions and the final diagnosis. There was no significant difference between EBUS and fluoroscopy. As expected, lesion size influenced the yield and details are listed in table 3 .

Table 1.-Established diagnosis in all patients

\begin{tabular}{|c|c|c|c|}
\hline \multirow[t]{2}{*}{ Diagnosis } & \multirow[t]{2}{*}{ n $(\%)$} & \multicolumn{2}{|c|}{ Diagnosis made by: } \\
\hline & & $\begin{array}{l}\text { EBUS } \\
\text { patients } \mathrm{n}\end{array}$ & $\begin{array}{c}\text { TBBX } \\
\text { patients } n\end{array}$ \\
\hline $\begin{array}{l}\text { Squamous cell } \\
\text { carcinoma }\end{array}$ & $16(32)$ & 13 & 14 \\
\hline Adeno carcinoma & $22(44)$ & 18 & 17 \\
\hline $\begin{array}{l}\text { Large cell } \\
\text { carcinoma }\end{array}$ & $1(2)$ & 1 & 0 \\
\hline Metastatic disease & $3(6)$ & 2 & 2 \\
\hline $\begin{array}{l}\text { Small cell } \\
\text { carcinoma }\end{array}$ & $3(6)$ & 2 & 2 \\
\hline Sarcoidosis & $2(4)$ & 2 & 2 \\
\hline Tuberculosis & $2(4)$ & 1 & 1 \\
\hline Aspergilloma & $1(2)$ & 1 & 0 \\
\hline
\end{tabular}

EBUS: endobronchial ultrasound; TBBX: transbronchial biopsy. Diagnosis not made by bronchoscopic technique was obtained by surgical biopsy.

Table 2.- Location of lesions

\begin{tabular}{lccc}
\hline & $n(\%)$ & \multicolumn{2}{c}{$\begin{array}{c}\text { Number of diagnosis } \\
\text { obtained by: }\end{array}$} \\
\cline { 3 - 4 } & & EBUS & TBBX \\
\hline Right upper lobe & $27(54)$ & 23 & 24 \\
Middle lobe & $4(8)$ & 3 & 2 \\
Right lower lobe & $2(4)$ & 1 & 0 \\
Left upper lobe & $11(22)$ & 9 & 9 \\
Left lower lobe & $6(12)$ & 4 & 3 \\
\hline
\end{tabular}

EBUS: endobronchial ultrasound; TBBX: transbronchial biopsy. 
Table 3.-Lesion size and diagnostic yield for both bronchoscopic biopsy techniques

\begin{tabular}{lcc}
\hline & Lesion $<3 \mathrm{~cm}$ & Lesion $>3 \mathrm{~cm}$ \\
\hline Patients n & 21 & 29 \\
EBUS n (\%) & $17(80)$ & $23(79)$ \\
Fluoroscopic n (\%) & $12(57)$ & $26(89)$ \\
\hline
\end{tabular}

Lesions measured by longest diameter. EBUS: endobronchial ultrasound.

Furthermore, there was no difference in diagnostic yield when analysing patient subgroups by age, sex or smoking habits.

In five patients $(10 \%)$, the histology was benign, and in $45(90 \%)$, the specimens were malignant (table 2 ). In nine patients $(18 \%)$, the diagnosis obtained by bronchoscopy saved a surgical procedure (sarcoidosis [2], tuberculoses [2], infection [1], metastatic disease [1] and small-cell lung cancer [3]).

Self-limited bleeding was observed in two cases. Severe bleeding was not observed in this study. One patient developed a pneumothorax $(2 \%)$, which was treated by tube thoracostomy. No deaths occurred with the diagnostic procedures.

\section{Discussion}

The most common bronchoscopic procedure for patients with peripheral nodules is fluoroscopy-guided TBBX [2]. With this technique the diagnostic yield strongly depends on the size of the lesion and varies between $20-75 \%[2,7]$. Additionally, there is radiation exposure to patients and staff. These shortcomings lead to frequent use of primary surgical biopsy procedures.

In order to save patients unnecessary surgery and to improve the diagnostic yield of endoscopic procedures, new imaging and guidance technology is needed.

To the best of the authors' knowledge, this is the first prospective study on the application of EBUS in peripheral lesions of the lung.

EBUS allows detailed imaging of the multiple layers of the bronchial wall and the parabronchial structures [7] and may also be helpful in guiding TBNA. As air functions like an insulator to ultrasonic waves [8], potential for EBUS-guided examination in the peripheral lung seemed limited when this imaging modality was introduced. When performing EBUS in the periphery, the present authors noted a distinct difference when introducing the probe into solid tumour compared to the air-filled alveoli. This encouraged the authors to perform this feasibility study.

Interestingly, the same yield could be obtained by EBUS-guided as compared to fluoroscopy-guided TBBX in this early study; and even though statistically not significant, the yield for lesions $<3 \mathrm{~cm}$ in size tended to be better than with fluoroscopy. For larger lesions, EBUS was not quite as good, but these results are tainted by the inability to localise several of those lesions in the right upper lobe. Interestingly, this high yield for EBUS-guided TBBX could be achieved even though the probe had to be removed before inserting the biopsy forceps. One could speculate that different set-ups allowing for imaging with EBUS while obtaining a biopsy could significantly enhance endoscopic biopsy success.

EBUS-guided TBBX is simple to perform and does not require more time than the procedure performed with fluoroscopy ( $\sim 6 \mathrm{~min}$ for each in this study). Obviously, a learning curve for EBUS interpretation is a fact and the present authors have found that it is necessary to perform 40-50 EBUS procedures to become comfortable.

The authors are aware that there is a limitation to the investigation, as both methods were applied in the same patient even if sequentially at random. Bias cannot be excluded as the location of the lesion may have been established by the respective first method. This bias was minimised by a crossover design in this study. The authors do not feel that prior fluoroscopy enhanced the ability to locate lesions by EBUS, as evidenced by the fact that three out of four lesions not found with EBUS were previously detected by fluoroscopy.

In summary, the result of this feasibility study shows that histological diagnosis of solitary pulmonary nodules and peripheral lesions of the lung by transbronchial biopsy can be achieved efficiently by endobronchial ultrasound guidance for transbronchial biopsy without the need for radiological equipment and radiation exposure. Further studies may be helpful to establish a firm role for endobronchial ultrasound guidance for transbronchial biopsy in bronchoscopic procedures.

\section{References}

1. Torrington KG, Kern JD. The utility of fiberbronchoscopy in the evaluation of solitary pulmonary nodules. Chest 1993; 104: 1021-1024.

2. Gasparini S, Ferretti M, Bichi Secchi E, Baldelli S, Zuccatosa L, Gusella P. Integration of transbronchial and percutaneous approach in the diagnosis of peripheral pulmonary nodules or masses. Experience with 1027 cases. Chest 1995; 108: 131-137.

3. Baaklini WA, Reinoso MA, Gorin AB, Sharafkaneh A, Manian P. Diagnostic yield of fiberoptic bronchoscopy in evaluating solitary pulmonary nodules. Chest 2000; 117: 1049-1054.

4. Herth F, Becker HD. Endobronchial ultrasound (EBUS) - assessment of a new diagnostic tool in bronchoscopy. Onkologie 2001; 24: 151-155.

5. Wang KP. Staging of bronchogenic carcinoma by bronchoscopy. Chest 1994; 106: 588-593.

6. Shannon JJ, Bude RO, Orens JB, et al. Endobronchial ultrasound-guided needle aspiration of mediastinal adenopathy. Am J Respir Crit Care Med 1996; 153: $1424-1430$

7. Lillington GA. Management of solitary pulmonary nodules. Dis Mon 1991; 37: 271-318.

8. Kremkau FW, Taylor KLW. Artifacts in ultrasound imaging. J Ultrasound Med 1986; 5: 227-237. 